\title{
Interpretation of Neutral Charm Mesons near Threshold as Unparticles
}

\author{
Eric Braaten \\ Department of Physics, The Ohio State University, Columbus, Ohio 43210, USA \\ Hans-Werner Hammer@ \\ Department of Physics, Technische Universität Darmstadt, 64289 Darmstadt, Germany \\ and ExtreMe Matter Institute EMMI and Helmholtz Forschungsakademie Hessen für FAIR (HFHF), \\ GSI Helmholtzzentrum für Schwerionenforschung GmbH, 64291 Darmstadt, Germany
}

(Received 13 July 2021; revised 7 November 2021; accepted 24 December 2021; published 20 January 2022)

\begin{abstract}
The existence of the $X(3872)$ resonance extremely close to the $D^{* 0} \bar{D}^{0}$ threshold implies that neutral charm mesons have an approximate nonrelativistic conformal symmetry. Systems consisting of these mesons with small kinetic energies produced in a short-distance reaction are unparticles in the sense that they can be created by operators with definite scaling dimensions in a nonrelativistic conformal field theory. There is a scaling region in which their energy distribution has power-law behavior with an exponent determined by the scaling dimension of the operator. The unparticle associated with two neutral charm mesons produces a peak in the recoil momentum spectrum of $K^{ \pm}$in inclusive decays of $B^{ \pm}$that has been observed. The scaling dimensions of the unparticles associated with three neutral charm mesons are calculated. They can be determined experimentally by measuring the invariant mass distributions for $X D^{0}$ or $X D^{* 0}$ in inclusive prompt production at the Large Hadron Collider.
\end{abstract}

DOI: 10.1103/PhysRevLett.128.032002

Introduction.-An elementary particle can be defined to be an irreducible representation of the Poincaré group. The concept of an "unparticle" was introduced by Georgi [1]. An unparticle is a system created by a local operator with a definite scaling dimension in a conformal field theory. It can therefore be defined to be an irreducible representation of the conformal symmetry group. The conformal group on 3+1-dimensional Minkowski space-time is a 15dimensional group that includes the Poincaré group and scale transformations as subgroups. A relativistic unparticle is characterized by a single number: the scaling dimension $\Delta$ of the operator. If the conformal field theory belongs to a hidden sector beyond the standard model of particle physics, the unparticle cannot be observed directly. However, it can be observed indirectly through the distribution of standard model particles produced in association with the unparticle [1]. There are aspects of the distribution determined by $\Delta$. The existence of unparticles in a hidden sector would produce novel signals in highenergy colliders [2-4]. The CMS Collaboration has searched for signals of unparticles in $p p$ collisions at the Large Hadron Collider (LHC) [5-7].

Published by the American Physical Society under the terms of the Creative Commons Attribution 4.0 International license. Further distribution of this work must maintain attribution to the author(s) and the published article's title, journal citation, and DOI. Funded by SCOAP ${ }^{3}$.
Hammer and Son recently pointed out that unparticles can also arise in nonrelativistic physics [8]. The nonrelativistic conformal symmetry group (also called the Schrödinger group) on 3+1-dimensional Galilean space-time is a 13-dimensional group that includes the Galilean group and scale transformations as subgroups. A nonrelativistic conformal field theory is a field theory with the nonrelativistic conformal symmetry [9], and a "nonrelativistic unparticle" is a system created by a local operator with a definite scaling dimension in such a theory. In contrast to the relativistic case, a nonrelativistic unparticle is characterized by two numbers: its mass $M$ and the scaling dimension $\Delta$ of the operator [8].

A physical realization of nonrelativistic unparticles is neutrons with small relative momenta produced by a shortdistance reaction [8]. Neutrons have a negative scattering length $a$ that is much larger than their effective range. A system of low-energy neutrons therefore has a scaling region in which their behavior is approximately scale invariant. In the unitary limit $1 / a \rightarrow 0$, their low-energy behavior can be described by a nonrelativistic conformal field theory. A system of $N$ neutrons created by a local operator with scaling dimension $\Delta_{N}$ is an unparticle. Its mass is $N m_{n}$, where $m_{n}$ is the kinetic mass of the neutron. For the two-neutron unparticle, the lowest scaling dimension is $\Delta_{2}=2$. For the three-neutron unparticle, the lowest scaling dimension is $\Delta_{3}=4.27272$.

The $N$-neutron unparticle can be created by a shortdistance nuclear reaction of the form $A_{1}+A_{2} \rightarrow$ $B+(n n \ldots)$ [8]. The invariant energy $E$ of the $N$ neutrons, 
which is their total kinetic energy in their center-ofmomentum (CM) frame, can be determined by measuring the momentum of the recoiling nucleus $B$. There is a scaling region of $E$ in which the differential cross section has the power-law behavior $E^{\Delta_{N}-5 / 2} d E$. The naive prediction for $N$ noninteracting particles is $E^{(3 N-5) / 2} d E$. The nontrivial power-law behavior is the smoking gun for an unparticle.

In this Letter, we point out that systems consisting of the neutral charm mesons $D^{0}, D^{* 0}, \bar{D}^{0}$, and $\bar{D}^{* 0}$ with small relative momenta produced by a short-distance reaction are unparticles. Their unparticle nature arises from the existence of the $X(3872)$ resonance extremely close to the threshold in the $J^{P C}=1^{++}$channel of $D^{* 0} \bar{D}^{0}$ and $D^{0} \bar{D}^{* 0}$. The $X(3872)$ resonance was discovered by the Belle Collaboration in 2003 [10]. Its quantum numbers were determined by the LHCb Collaboration in 2013 to be $J^{P C}=1^{++}[11]$. The most precise measurements of the mass by the LHCb Collaboration give an energy relative to the $D^{* 0} \bar{D}^{0}$ threshold of $\varepsilon_{X}=-0.07 \pm 0.12 \mathrm{MeV}[12,13]$, which implies $\left|\varepsilon_{X}\right|<0.22 \mathrm{MeV}$ at the $90 \%$ confidence level. The quantum numbers and the tiny value of $\varepsilon_{X}$ imply that the wave function of $X$ at long distances is that of a charm-meson molecule with the flavor structure $\left(D^{* 0} \bar{D}^{0}+D^{0} \bar{D}^{* 0}\right) / \sqrt{2}$. The wave function at short distances is unknown, but the possibilities include the $\chi_{c 1}(2 P)$ charmonium state and a compact tetraquark with constituents $c \bar{c} q \bar{q}$ (see, e.g., Ref. [14]). If $\varepsilon_{X}>0$, then $X$ is a virtual state like the dineutron, in which case the unparticle physics of neutral charm mesons is qualitatively like that of neutrons. We assume that $\varepsilon_{X}<0$ so that $X$ is a bound state, in which case the unparticle physics of neutral charm mesons exhibits qualitatively new features. A peak from the two-charm-meson unparticle has been observed in inclusive $B$ meson decays. We calculate the nontrivial scaling dimensions for the three-charm-meson unparticles, and we explain how they can be determined experimentally at the LHC.

Two-charm-meson unparticle.-The LHCb Collaboration has analyzed the line shape of $X(3872)$ in the $J / \psi \pi^{+} \pi^{-}$ channel using a Flatte-inspired amplitude [12]. In addition to the pole for $X$ near the $D^{* 0} \bar{D}^{0}$ threshold, their fitted amplitude has a second pole about $3.6 \mathrm{MeV}$ below the threshold. We adopt $\varepsilon_{0}=3.6 \mathrm{MeV}$ as an estimate of the energy scale associated with the range of interactions between charm mesons. The behavior of a system of neutral charm mesons whose kinetic energies in their $\mathrm{CM}$ frame are all in the scaling region between $\left|\varepsilon_{X}\right|$ and $\varepsilon_{0}$ is approximately scale invariant. In the limit $\varepsilon_{X} \rightarrow 0$, their low-energy behavior can be described by a nonrelativistic conformal field theory. A system of neutral charm mesons created by a local operator with definite scaling dimension in this field theory is an unparticle. For the operator that creates charm mesons in the resonant $C=+$ channel $\left(D^{* 0} \bar{D}^{0}+D^{0} \bar{D}^{* 0}\right) / \sqrt{2}$, the lowest scaling (a)
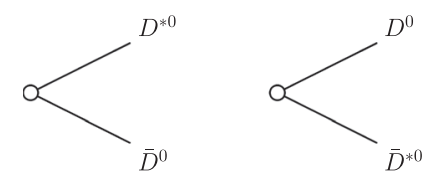

(b)

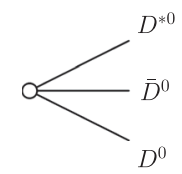

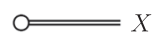

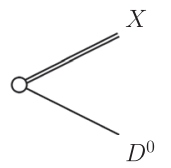

FIG. 1. (a) The creation of the $X$ unparticle at a point produces $D^{* 0} \bar{D}^{0}$ and $D^{0} \bar{D}^{* 0}$ scattering states and the $X(3872)$ bound state. (b) The creation of the $X D$ unparticle produces $D^{0} D^{* 0} \bar{D}^{0}$, $D^{0} D^{0} \bar{D}^{* 0}$, and $D^{0} X(3872)$. Charm mesons and $X(3872)$ are shown by single and double lines, respectively.

dimension is $\Delta_{2}=2$. We refer to the system created by this operator, which consists of $D^{* 0} \bar{D}^{0}$ and $D^{0} \bar{D}^{* 0}$ scattering states and also the $X(3872)$ resonance, as the " $X$ unparticle" [see Fig. 1(a)]. The corresponding scaling dimension for the $C=-$ channel $\left(D^{* 0} \bar{D}^{0}-D^{0} \bar{D}^{* 0}\right) / \sqrt{2}$ is three, twice the scaling dimension $3 / 2$ of an operator that creates a single nonrelativistic particle. The smoking gun for the $X$ unparticle is power-law behavior determined by the scaling dimension $\Delta_{2}=2$.

Given any exclusive reaction that produces $X(3872)$ and a single recoiling particle with relative momentum much larger than $\sqrt{2 M_{D} \varepsilon_{0}}$, the $X$ unparticle can be observed by measuring the momentum distribution of that particle in the corresponding inclusive reaction. The $X$ unparticle appears as a narrow peak in the momentum distribution related by kinematics to a peak near $3872 \mathrm{MeV}$ in the invariant mass distribution of the other particles. An example is the inclusive decay $B^{ \pm} \rightarrow K^{ \pm}+$anything. The $B A B A R$ Collaboration has collected a sample of fully reconstructed $B^{ \pm}$events in $e^{+} e^{-}$annihilation at the $\Upsilon(4 S)$ resonance and then measured the momentum of a $K^{ \pm}$in the events [15]. In addition to peaks in the momentum distribution associated with known charmonium states, there is a significant peak near $1141 \mathrm{MeV}$ corresponding to recoil against a system with invariant mass near $3872 \mathrm{MeV}$. This peak can be identified with the $X$ unparticle.

In the decay of $B^{ \pm}$, the unparticle nature of the system recoiling against the $K^{ \pm}$is reflected in the invariant mass distribution of the threshold enhancement in $D^{* 0} \bar{D}^{0}$ and $D^{0} \bar{D}^{* 0}$. The production rates from the creation of the $X$ unparticle at short distances can be calculated in an effective field theory in which the only interaction between charm mesons is a contact interaction that gives a large scattering length $a$ in the channel with the $X$ resonance. If the $X(3872)$ is a bound state, $a$ is positive and the energy of $X(3872)$ relative to the $D^{* 0} \bar{D}^{0}$ threshold is $\varepsilon_{X}=-1 /\left(2 \mu a^{2}\right)$, where $\mu$ is the reduced mass of $D^{* 0} \bar{D}^{0}$. The production rate $R_{X}$ of $X(3872)$ is the product of $\left|\varepsilon_{X}\right|^{1 / 2}$ and a short-distance factor. The differential production rate of $D^{* 0} \bar{D}^{0}$ is the product of 


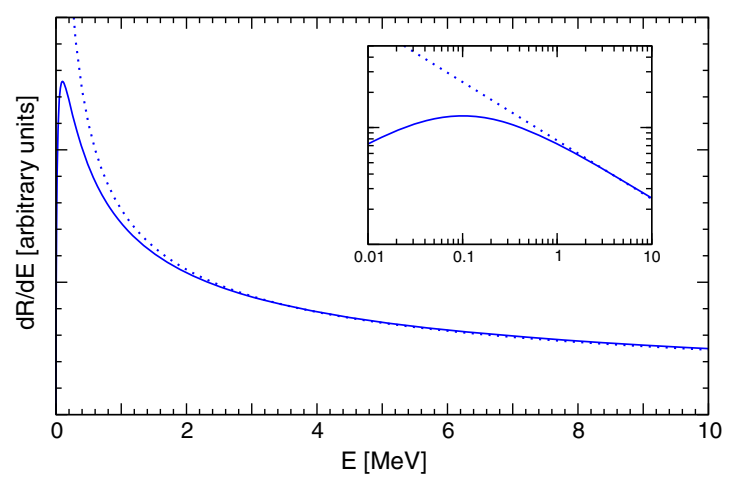

FIG. 2. Production rate $d R / d E$ for $D^{* 0} \bar{D}^{0}$ from the creation of neutral charm mesons at short distances as a function of the invariant energy $E$ (solid line) for $\varepsilon_{X}=-0.1 \mathrm{MeV}$. The dotted line shows the $E^{-1 / 2}$ scaling behavior. The inset is the same plot on a $\log -\log$ scale.

the same short-distance factor and a function of the invariant energy $E$ of the charm mesons [16]. If $E \ll \varepsilon_{0}$, the production rate can be expressed as

$$
d R_{D^{* 0} \bar{D}^{0}}=\frac{R_{X} \sqrt{E}}{4 \pi\left|\varepsilon_{X}\right|^{1 / 2}\left(\left|\varepsilon_{X}\right|+E\right)} d E .
$$

As shown in Fig. 2, $d R / d E$ increases from zero at the $D^{* 0} \bar{D}^{0}$ threshold to a peak near $\left|\varepsilon_{X}\right|$, and then it decreases. There is a scaling region between $\left|\varepsilon_{X}\right|$ and $\varepsilon_{0}$ in which $d R / d E$ decreases with the power-law behavior $E^{\Delta_{2}-5 / 2}=E^{-1 / 2}$. Beyond the scaling region, there is a crossover to an increasing production rate, so $d R / d E$ has a local minimum at an energy of order $\varepsilon_{0}$. (This crossover is not shown in Fig. 2.) The unparticle nature can be verified by observing the power-law behavior in the scaling region. The $B A B A R$ experiment did not have enough data to resolve the structure of the peak associated with the $X$ unparticle, but the powerlaw behavior may be observable in the much larger datasets that will be collected by the Belle II experiment.

The prompt production of $X(3872)$ at a hadron collider is the contribution that does not come from the weak decay of a $b$ hadron. It is dominated by the creation of a charm quark-antiquark pair that evolves at short distances into two charm mesons. If the two charm mesons are neutral and have small relative momentum, they can form the $X$ unparticle. The $X(3872)$ component of the $X$ unparticle can be observed at a hadron collider through its decay into $J / \psi \pi^{+} \pi^{-}$, because the decay $J / \psi \rightarrow \mu^{+} \mu^{-}$provides a trigger. Cross sections for the inclusive production of $X(3872)$ in $p p$ collisions at the LHC have been measured by the LHCb and CMS Collaborations $[17,18]$. The $D^{* 0} \bar{D}^{0}$ and $D^{0} \bar{D}^{* 0}$ components of the $X$ unparticle cannot be easily observed at a hadron collider.

Three-charm-meson unparticles.-We now consider unparticles associated with three neutral charm mesons. They consist of scattering states of three charm mesons and scattering states of $X$ and one charm meson. We refer to the unparticle consisting of $D^{0} D^{* 0} \bar{D}^{0}, D^{0} D^{0} \bar{D}^{* 0}$, and $D^{0} X$ as the $X D$ unparticle [see Fig. 1(b)] and the unparticle consisting of $D^{* 0} D^{* 0} \bar{D}^{0}, D^{* 0} D^{0} \bar{D}^{* 0}$, and $D^{* 0} X$ as the $X D^{*}$ unparticle.

Systems consisting of three low-energy neutral charm mesons have been studied previously by Canham et al. [19]. They calculated the low-energy differential cross sections for the scattering of $D^{0} X$ and $D^{* 0} X$ in an effective field theory in which the charm mesons have a large scattering length $a$ in the channel with the $X$ resonance. They solved the Skorniakov-Ter-Martirosian (STM) [20] integral equation for partial-wave scattering amplitudes as functions of the kinetic energy $E$. The $\mathrm{S}$-wave scattering lengths for $D^{0} X$ and $D^{* 0} X$ are equal to $a$ multiplied by a large negative coefficient: $a_{D^{0} X}=-9.7 a, a_{D^{* 0} X}=-16.6 a$. Thus the threshold cross sections $4 \pi a_{D X}^{2}$ are more than 2 orders of magnitude larger than the threshold cross section $2 \pi a^{2}$ for $D^{* 0} \bar{D}^{0}$ scattering.

The scaling dimensions of the operators that create the $X D$ and $X D^{*}$ unparticles can be determined from the homogeneous form of the S-wave STM equation in Ref. [19]. The S-wave STM equation is an integral equation for the amplitude $T_{0}(k, p)$ for the scattering of $D X$ (where $D$ is $D^{0}$ or $D^{* 0}$ ) from relative momentum $p$ to relative momentum $k$ with off-shell energy $E$. The homogeneous $\mathrm{S}$-wave STM equation reduces in the limits $E \rightarrow 0, \varepsilon_{X} \rightarrow 0$ to

$T_{0}(k, p)=\int_{0}^{\infty} d q \frac{T_{0}(k, q)}{4 \pi r \sqrt{1-r^{2}} p} \log \frac{p^{2}+q^{2}+2 r p q}{p^{2}+q^{2}-2 r p q}$,

where $r=1 /\left(1+M_{D^{* 0}} / M_{D^{0}}\right)=0.48166$ if $D$ is $D^{0}$ and $r=1 /\left(1+M_{D^{0}} / M_{D^{* 0}}\right)=0.51834$ if $D$ is $D^{* 0}$. The condition for a solution with the power-law behavior $T_{0}(k, p)=p^{s-1}$ can be derived by inserting this ansatz into Eq. (2) and evaluating the integral over $q$,

$$
\sin [s \arcsin (r)]=2 r \sqrt{1-r^{2}} s \cos (s \pi / 2) .
$$

The smallest positive solution for $s$ is 0.60119 if $D$ is $D^{0}$ and 0.58697 if $D$ is $D^{* 0}$. The scaling dimension $\Delta$ of the operator that creates the unparticle is $\Delta=s+5 / 2$. The $X D$ unparticle has mass $M_{D^{* 0}}+2 M_{D^{0}}$ and scaling dimension $\Delta_{3}=3.10119$. The $X D^{*}$ unparticle has mass $2 M_{D^{* 0}}+$ $M_{D^{0}}$ and scaling dimension $\Delta_{3 *}=3.08697$.

The solutions to the STM equation in Ref. [19] can be extended out to the scaling region of the total kinetic energy $E$ of $X D^{0}$ or $X D^{* 0}$ beyond the $X$ breakup thresholds. The relative $\mathrm{S}$-, $\mathrm{P}$-, and $\mathrm{D}$-wave contributions to the $D^{0} X$ and $D^{* 0} X$ cross sections are shown as functions of $E$ in Fig. 3 . There is a scaling region at large $E$ where the cross sections for $D^{0} X$ and $D^{* 0} X$ both scale as $E^{-1.6}$. It is difficult to resolve the differences between the exponents for $D^{0} X$ and 


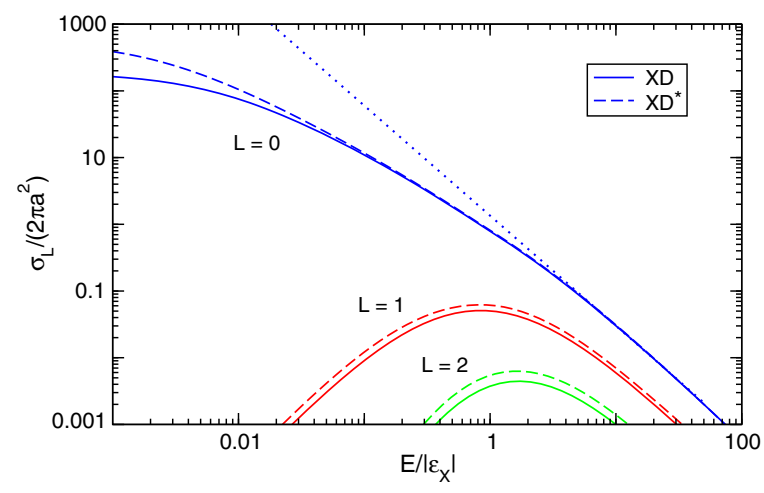

FIG. 3. Partial-wave cross sections $\sigma_{L}$ for $D^{0} X$ (solid curves) and $D^{* 0} X$ (dashed curves) as functions of the kinetic energy $E$ for $L=0,1,2$. The dotted line shows the $E^{-1.6}$ scaling behavior.

$D^{* 0} X$ numerically. The exponents are presumably determined by the scaling dimensions $\Delta_{3}$ and $\Delta_{3 *}$, but we have not succeeded in determining them analytically.

The $X D$ and $X D^{*}$ unparticles could be observed at a future $e^{+} e^{-}$collider operating at an energy just above the threshold for $B_{c}^{+} B_{c}^{-}$at $12.55 \mathrm{GeV}$. If a sample of fully reconstructed $B_{c}^{ \pm}$events is collected, the unparticle could be observed through the momentum distribution of $\pi^{ \pm}$or $K^{ \pm}$in the events. The $X D$ and $X D^{*}$ unparticles determine the invariant mass distribution of the recoiling system near the $D^{0} D^{* 0} \bar{D}^{0}$ threshold at $5.737 \mathrm{GeV}$ and near the $D^{* 0} D^{* 0} \bar{D}^{0}$ threshold at $5.879 \mathrm{GeV}$. There is a scaling region of the invariant energy $E$ relative to the threshold where the distribution has power-law behavior: $E^{\Delta_{3}-5 / 2} d E=E^{0.6012} d E$ for the $X D$ unparticle and $E^{\Delta_{3 *}-5 / 2} d E=E^{0.5870} d E$ for the $X D^{*}$ unparticle. The rate of increase is much smaller than the naive prediction $E^{2} d E$ for three noninteracting particles.

The prompt production of $X(3872)$ at a hadron collider must include a small contribution from the creation of two charm quark-antiquark pairs that evolve at short distances into four charm mesons. If three of the charm mesons are neutral and have small relative momenta, they can form the $X D$ or $X D^{*}$ unparticle. The $D^{0} X$ and $D^{* 0} X$ components of these unparticles can be observed at a hadron collider through the decay of $X(3872)$ into $J / \psi \pi^{+} \pi^{-}$, because the decay $J / \psi \rightarrow \mu^{+} \mu^{-}$provides a trigger. The amplitude for producing $D X$ with kinetic energy $E$ from the creation of a three-charm-meson unparticle at a point can be calculated by solving the STM equation with a constant inhomogeneous term $g_{X D}$,

$$
\begin{aligned}
\Gamma(p, E)= & g_{X D}+\int_{0}^{\infty} \frac{d q q}{2 \pi r p} Q_{0}\left(\frac{p^{2}+q^{2}-2 \mu E+1 / a^{2}}{2 r p q}\right) \\
& \times \frac{\Gamma(q, E)}{-1 / a+\left[\left(\mu / \mu_{X D}\right) q^{2}-2 \mu E+1 / a^{2}\right]^{1 / 2}}
\end{aligned}
$$

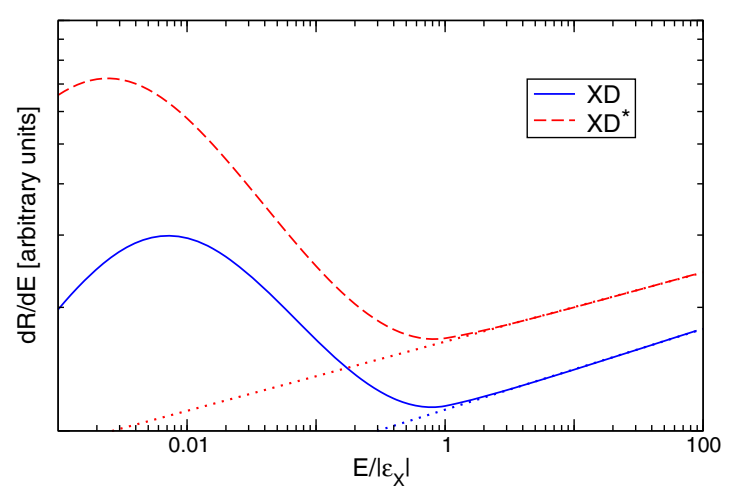

FIG. 4. Production rates $d R / d E$ for $D^{0} X$ (solid curve) and $D^{* 0} X$ (dashed curve) from the creation of neutral charm mesons at short distances as functions of the invariant energy $E$. The dotted lines show the $E^{+0.08}$ scaling behavior.

where $Q_{0}(z)=\frac{1}{2} \log [(z+1) /(z-1)]$ and $\mu_{X D}$ is the $X D$ reduced mass. The STM equation is solved with $E$ replaced by $E+i \epsilon$ in the limit $\epsilon \rightarrow 0^{+}$. The amplitude $\Gamma(p, E)$ is put on shell by setting $p=\left(2 \mu_{D X} E\right)^{1 / 2}$. The production rate $d R / d E$ can then be obtained from $|\Gamma|^{2}$ integrated over phase space. For $E$ extremely close to the $D X$ threshold, $d R / d E$ is determined by the tiny energy scale $\varepsilon_{D X}=$ $1 /\left(2 \mu_{D X} a_{D X}^{2}\right)$, which is $\varepsilon_{D^{0} X}=0.82$ or $\varepsilon_{D^{* 0} X}=0.26 \mathrm{keV}$. As shown in Fig. 4, $d R / d E$ increases from zero at the threshold to a peak near $\varepsilon_{D X}$, and it then decreases to a local minimum at an energy of order $\left|\varepsilon_{X}\right|$. Beyond the minimum, there is a scaling region where $d R / d E$ increases with a power-law behavior. The power-law behavior of the amplitudes $\Gamma$ for $D^{0} X$ and $D^{* 0} X$ are both determined numerically to be $E^{-0.21}$, with an error in the last digit of the exponent. There is a crossover to a more rapidly increasing production rate at an energy of order $\varepsilon_{0}$. (This crossover is not shown in Fig. 4.)

The power-law behavior in the scaling region can be determined from the general analytic result for the threepoint Green's function in coordinate space for primary operators in a nonrelativistic conformal field theory. We take the three operators to be an operator with scaling dimension $\Delta_{3}$ (or $\Delta_{3 *}$ ) and mass $M_{3}=M_{1}+M_{2}$, an operator with scaling dimension $\Delta_{2}=2$ and mass $M_{2}$, and a single-particle operator with scaling dimension $\Delta_{1}=$ $3 / 2$ and mass $M_{1}$. The three-point function was first deduced by Henkel and Unterberger from the three-point function for a relativistic conformal field theory in two higher dimensions [21]. It has also been obtained by Fuertes and Moroz [22] and by Volovich and Wen [23] using holography and the AdS/CFT correspondence. The Fourier transform of the three-point function has a pole in the energy $E_{1}$ of the single particle. We take the total energy in the CM frame to be $E$ and the momentum of the particle to be $p$. The residue of the pole at $E_{1}=p^{2} /\left(2 M_{1}\right)$ is 


$$
\begin{aligned}
G(E, p)= & C \int_{0}^{1} d x x^{\Delta_{3}^{12} / 2-1}(1-x)^{\Delta_{2}^{13} / 2-1}\left(1+r_{12} x\right)^{3 / 2} \\
& \times\left[(1-x) p^{2} /\left(2 M_{12}\right)-\left(1+r_{12} x\right) E\right]^{\left(\Delta_{1}^{23}-5\right) / 2},
\end{aligned}
$$

where $\Delta_{k}^{i j}=\Delta_{i}+\Delta_{j}-\Delta_{k}, \quad M_{12}=M_{1} M_{2} /\left(M_{1}+M_{2}\right)$, $r_{12}=M_{1} / M_{2}$, and $C$ is a constant. The amplitude for producing the unparticle and the single particle is obtained by dividing by the unparticle propagator $\left[E-E_{1}-p^{2} /\right.$ $\left.\left(2 M_{2}\right)\right]^{\Delta_{2}-5 / 2}$. The amplitude $\Gamma$ for producing $X(3872)$ and a single charm meson with total energy $E \gg\left|\varepsilon_{X}\right|$ is then obtained by taking the limit $p \rightarrow\left(2 M_{12} E\right)^{1 / 2}$ and multiplying by $\left|\varepsilon_{X}\right|^{-1 / 2}$. This amplitude is determined by the integral in Eq. (5) near the lower end point of $x$. Its scaling behavior is $E^{-\Delta_{3}^{12} / 2}=E^{\left(\Delta_{3}-7 / 2\right) / 2}$. Our analytic predictions for the exponents are -0.1994 for $D^{0} X$ and -0.2065 for $D^{* 0} X$. Both are consistent with our numerical result -0.21 for the exponent for $\Gamma$ from the solutions to the STM equation (4). Figure 4 shows the production rate $d R / d E$, which is given by $|\Gamma|^{2}$ times a phase space factor $\sqrt{E}$, leading to $E^{0.08}$ scaling. The conformal field theory prediction for $d R / d E$ is $E^{\Delta_{3}-3}=E^{0.1012}$ for $D^{0} X$ and $E^{\Delta_{3 *}-3}=E^{0.0870}$ for $D^{* 0} X$, compared to the naive prediction $E^{1 / 2}$ for two noninteracting particles.

Conclusion.-Nonrelativistic unparticles arise naturally in any system that can be described by a nonrelativistic field theory close to a conformally invariant limit. They can be used to identify reactions with power-law behavior characterized by nontrivial exponents. We have argued that systems of neutral charm mesons with small invariant energy are unparticles. We emphasize that unparticle physics occurs only in systems that do not exhibit the Efimov effect, because Efimov physics breaks the scale symmetry down to a discrete scale symmetry [24]. Unparticle physics can arise in systems of $D$ and $D^{*}$ mesons, because the attraction of three charm mesons is below the critical strength for the Efimov effect. It would be interesting to find other examples of nonrelativistic unparticles in nature. One could, for example, exploit the remarkable control of interactions that is possible with ultracold atoms [25] to engineer new systems with unparticles.

We thank Dam Thanh Son for discussions. The research of E. B. was supported in part by the U.S. Department of Energy under Award No. DE-SC0011726. H.-W. H. was supported by the Deutsche Forschungsgemeinschaft (DFG, German Research Foundation)—Project-ID 279384907SFB 1245 and by the German Federal Ministry of Education and Research (BMBF) (Grant No. 05P21RDFNB).

[1] H. Georgi, Unparticle Physics, Phys. Rev. Lett. 98, 221601 (2007).

[2] K. Cheung, W. Y. Keung, and T. C. Yuan, Collider Signals of Unparticle Physics, Phys. Rev. Lett. 99, 051803 (2007).
[3] H. Georgi, Another odd thing about unparticle physics, Phys. Lett. B 650, 275 (2007).

[4] K. Cheung, W. Y. Keung, and T.C. Yuan, Collider phenomenology of unparticle physics, Phys. Rev. D 76, 055003 (2007).

[5] V. Khachatryan et al. (CMS Collaboration), Search for dark matter, extra dimensions, and unparticles in monojet events in proton-proton collisions at $\sqrt{s}=8 \mathrm{TeV}$, Eur. Phys. J. C 75, 235 (2015).

[6] V. Khachatryan et al. (CMS Collaboration), Search for dark matter and unparticles produced in association with a $\mathrm{Z}$ boson in proton-proton collisions at $\sqrt{s}=8 \mathrm{TeV}$, Phys. Rev. D 93, 052011 (2016).

[7] A. M. Sirunyan et al. (CMS Collaboration), Search for dark matter and unparticles in events with a $Z$ boson and missing transverse momentum in proton-proton collisions at $\sqrt{s}=13 \mathrm{TeV}$, J. High Energy Phys. 03 (2017) 061.

[8] H.-W. Hammer and D. T. Son, Unnuclear physics, Proc. Natl. Acad. Sci. U.S.A. 118, e2108716118 (2021).

[9] Y. Nishida and D. T. Son, Nonrelativistic conformal field theories, Phys. Rev. D 76, 086004 (2007).

[10] S. K. Choi et al. (Belle Collaboration), Observation of a Narrow Charmonium-like State in Exclusive $B^{ \pm} \rightarrow$ $K^{ \pm} \pi^{+} \pi^{-} J / \psi$ Decays, Phys. Rev. Lett. 91, 262001 (2003).

[11] R. Aaij et al. (LHCb Collaboration), Determination of the X(3872) Meson Quantum Numbers, Phys. Rev. Lett. 110, 222001 (2013).

[12] R. Aaij et al. (LHCb Collaboration), Study of the lineshape of the $\chi_{c 1}(3872)$ state, Phys. Rev. D 102, 092005 (2020).

[13] R. Aaij et al. (LHCb Collaboration), Study of the $\psi_{2}$ (3823) and $\chi_{c 1}(3872)$ states in $B^{+} \rightarrow\left(J / \psi \pi^{+} \pi^{-}\right) K^{+}$decays, J. High Energy Phys. 08 (2020) 123.

[14] N. Brambilla, S. Eidelman, C. Hanhart, A. Nefediev, C. P. Shen, C.E. Thomas, A. Vairo, and C.Z. Yuan, The $X Y Z$ states: Experimental and theoretical status and perspectives, Phys. Rep. 873, 1 (2020).

[15] J. P. Lees et al. (BABAR Collaboration), Measurements of the Absolute Branching Fractions of $B^{ \pm} \rightarrow K^{ \pm} X_{c \bar{c}}$, Phys. Rev. Lett. 124, 152001 (2020).

[16] E. Braaten and M. Kusunoki, Factorization in the production and decay of the $X(3872)$, Phys. Rev. D 72, 014012 (2005).

[17] R. Aaij et al. (LHCb Collaboration), Observation of $X(3872)$ production in $p p$ collisions at $\sqrt{s}=7 \mathrm{TeV}$, Eur. Phys. J. C 72, 1972 (2012).

[18] S. Chatrchyan et al. (CMS Collaboration), Measurement of the $X(3872)$ production cross section via decays to $J / \psi \pi^{+} \pi^{-}$in $p p$ collisions at $\sqrt{s}=7 \mathrm{TeV}$, J. High Energy Phys. 04 (2013) 154.

[19] D. L. Canham, H.-W. Hammer, and R. P. Springer, On the scattering of $D$ and $D^{*}$ mesons off the $X(3872)$, Phys. Rev. D 80, 014009 (2009).

[20] G. V. Skorniakov and K. A. Ter-Martirosian, Three-body problem for short range forces 1 . Scattering of low energy neutrons by deuterons, Zh. Eksp. Teor. Fiz. 31, 775 (1956) [Sov. Phys. JETP 4, 648 (1957)].

[21] M. Henkel and J. Unterberger, Schrödinger invariance and space-time symmetries, Nucl. Phys. B660, 407 (2003). 
[22] C. A. Fuertes and S. Moroz, Correlation functions in the non-relativistic AdS/CFT correspondence, Phys. Rev. D 79, 106004 (2009).

[23] A. Volovich and C. Wen, Correlation functions in non-relativistic holography, J. High Energy Phys. 05 (2009) 087.
[24] E. Braaten and H.-W. Hammer, Universality in few-body systems with large scattering length, Phys. Rep. 428, 259 (2006).

[25] C. Chin, R. Grimm, P. Julienne, and E. Tiesinga, Feshbach resonances in ultracold gases, Rev. Mod. Phys. 82, 1225 (2010). 